\title{
Deriving myocardial blood flow reserve from perfusion datasets: Dream or reality?
}

\author{
Alexis Poitrasson-Rivière, PhD $\mathbb{D}^{\mathrm{a}}{ }^{\mathrm{a}}$ and Venkatesh L. Murthy, MD, PhD $\mathbb{\circledR}^{\mathrm{b}}$ \\ a INVIA Medical Imaging Solutions, Ann Arbor, MI \\ b Division of Cardiovascular Medicine, Department of Internal Medicine and Frankel \\ Cardiovascular Center, University of Michigan, Ann Arbor, MI
}

Received Dec 7, 2020; accepted Dec 8, 2020

doi: $10.1007 / \mathrm{s} 12350-020-02488-4$

\section{See related article, pp. 835-850}

\section{INTRODUCTION}

Although quantitative estimates of myocardial blood flow (MBF) and flow reserve (MFR) from positron emission tomography (PET) myocardial perfusion imaging (MPI) have widely recognized value, ${ }^{1-3}$ accurate estimates require high-quality scanners, as well as rigorous injection and optimized acquisition protocols. ${ }^{4}$ Dynamic series used for computation of MBF and MFR are prone to numerous quality issues including patient motion and scanner saturation. Although the former can be readily recognized and corrected, ${ }^{5-7}$ identification of saturation by inspection of dynamic series is not straightforward or reliable. ${ }^{8}$ Furthermore, obtaining data without saturation is near impossible with certain older scanners. Consequently, there is great interest in simpler alternatives to standard kinetic modeling approaches, ${ }^{9,10}$ which are robust to these issues. Toward this end, work by Juneau et al in the present issue of the Journal of Nuclear Cardiology investigates the estimation of MFR from the myocardial activity ratio (MAR) from static images. ${ }^{11}$

\footnotetext{
Supplementary Information The online version of this article (h ttps://doi.org/10.1007/s12350-020-02488-4) contains supplementary material, which is available to authorized users.

Reprint requests: Alexis Poitrasson-Rivière, PhD, INVIA Medical Imaging Solutions, Ann Arbor, MI; apoitrasson@inviasolutions. com

J Nucl Cardiol 2021;28:851-4.

$1071-3581 / \$ 34.00$

Copyright (C) 2021 American Society of Nuclear Cardiology.
}

\section{BACKGROUND}

Two methods of calculating MBF from PET MPI have been well validated: net retention modeling and compartmental modeling. Full compartmental modeling involves fitting observed dynamic data to a kinetic model describing the transit of tracer from the blood to tissue compartments (Figure 1A). The net retention method is a simplification of compartmental modeling wherein the tissue activity is scaled by activity in the blood pool (Figure 1B). The method proposed by Juneau et al is a further simplification of the net retention method wherein blood-pool activity is assumed to be the same at both rest and stress (Figure 1C). The authors have fit a non-linear regression formula to optimize the correlation between the activity ratio and measured MFR (which perhaps could have been done more robustly and simply by log transformation of the data). While this method avoids the need for acquisition of blood-pool phase images, it still requires a rigorous injection and acquisition protocol as the two images must result from injections of the same activity, identical delays between injection and acquisition, and identical acquisition times.

This method, first outlined more than 20 years ago, ${ }^{9,12}$ and applied to ${ }^{18} \mathrm{~F}$-flurpiridaz in $2011,{ }^{10}$ implicitly assumes that cardiac output (CO) does not change between rest and stress. Increase of $\mathrm{CO}$ at stress would result in more rapid clearance of tracer from the blood pool, violating this assumption. Juneau et al evaluated this assumption through multiple linear regression between MAR, MFR, and CO, as well as comparing $\mathrm{CO}$ ratios to liver uptake ratios for vasodilator stress testing. Results showed limited impact of CO on MAR calculations but require further investigation as to whether this applies to other populations and stress pharmaceuticals (dipyridamole was used in the study) and to exercise stress. Importantly, changes in ejection 

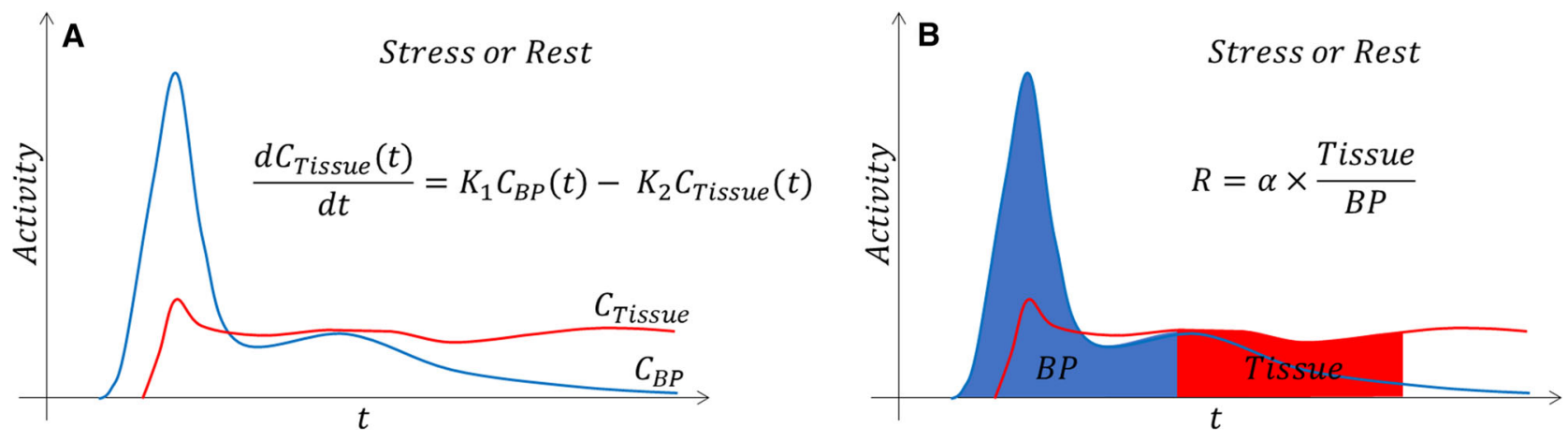

$$
\begin{gathered}
M B F \propto K_{1} \propto R \\
M F R=\frac{M B F_{\text {stress }}}{M B F_{\text {rest }}}
\end{gathered}
$$
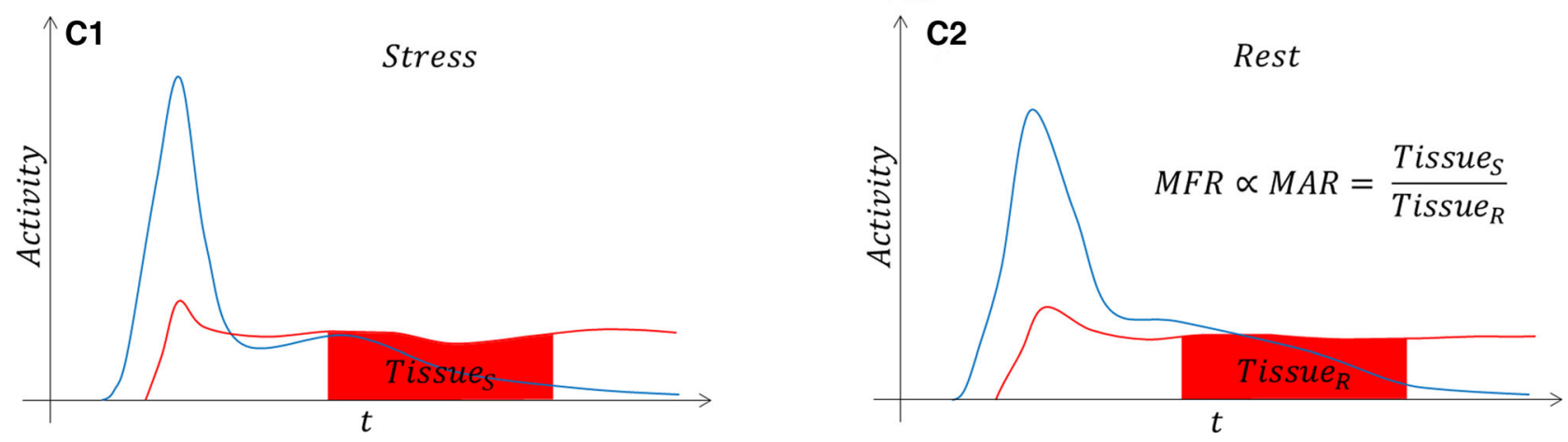

Figure 1. Simplified overview of methods used to derive MFR. (A) 1-tissue compartment modeling fits a kinetic model to the observed tissue (red) and blood (blue) time-activity curves from dynamic series. (B) The net retention model uses area under the blood time-activity curve during the blood-pool phase and area under the tissue time-activity curve during the tissue phase to simplify calculation. Higher cardiac output leads to more rapid clearance of the blood activity, and thus, a lower area under the curve. (C) In the MAR method used by Juneau et al the blood-pool activity is assumed to be unchanged between rest and stress so MFR can be estimated as the ratio of the activity in the tissue at stress divided by rest. Partial volume effects and spillover are left out of $\mathrm{A}$ and $\mathrm{B}$ for readability. Note that the area under the blue blood-pool time-activity curve is slightly smaller at stress than rest, accounting for a modest increase in cardiac output, resulting in faster clearance.

fraction and heart rate with stress have been shown to be prognostically important ${ }^{13,14}$ and related to the presence of $\mathrm{CAD},{ }^{15}$ one must wonder if the lack of relationship between $\mathrm{CO}$ and MAR was simply due to lack of power or technique.

\section{PROMISE AND LIMITATIONS}

The data presented by Juneau et al show a moderate correlation between measured MFR and estimated MFR from their simplified method with $R^{2}$ of 0.40 and 0.53 for ${ }^{13} \mathrm{NH}_{3}$ and ${ }^{82} \mathrm{Rb}$, respectively. This implies only about half or less of the information in the data can be retrieved by this method. Yet, the receiver-operating analysis yielded promising results with areas under the curve (AUC) of 0.832 and 0.904 , respectively, for ${ }^{13} \mathrm{NH}_{3}$ and ${ }^{82} \mathrm{Rb}$. Using estimated MFR to detect MFR $<2$ yielded a sensitivity of $73.3 \%$ and a specificity of $79.9 \%$ for ${ }^{13} \mathrm{NH}_{3}$ and $77.3 \%$ and $88 \%$ for ${ }^{82} \mathrm{Rb}$.

How can we rationalize that only half or less of the information in a properly measured MFR is obtained in these estimates and yet the sensitivity and specificity are reasonably good? First, these results may be optimistic due to a relatively low prevalence of patients with reduced MFR. However, more likely is that the sensitivity and specificity are over-optimistic due to unblinded remeasurement in the core lab, ${ }^{16}$ which will not be possible in real-world practice where the gold standard measurement is not available. To evaluate this, we simulated 10,000 hypothetical datasets for each tracer with the same sample size as in the Juneau et al study $(\mathrm{N}=250)$, using the observed mean and standard deviation of MFR and estimated MFR, and their correlation (square root of $R^{2}$ ) [scripts and output of 

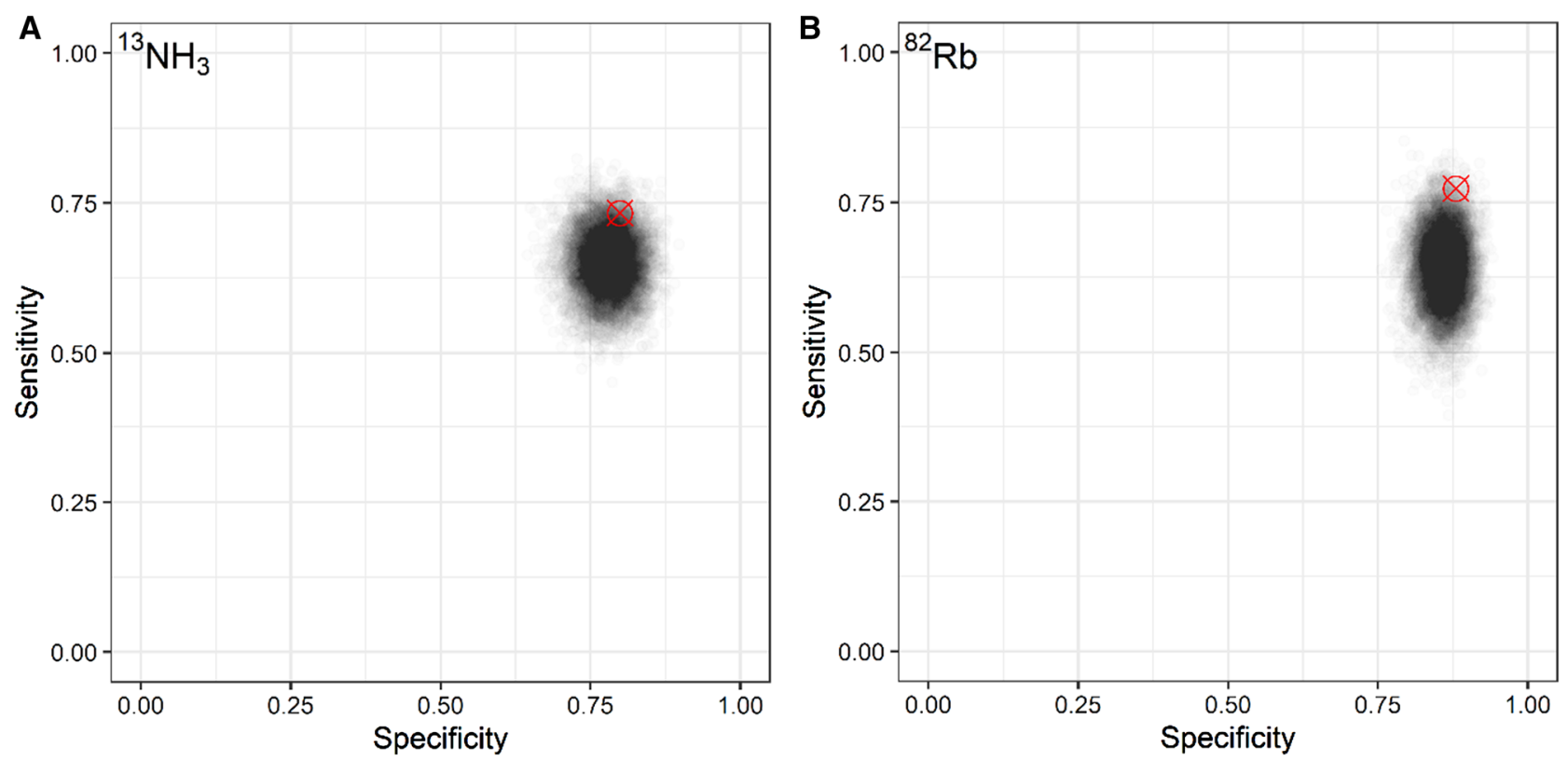

Figure 2. Scatter plots of the sensitivity and specificity derived from simulations of the study from Juneau et al for ${ }^{13} \mathrm{NH}_{3}$ (panel A) and ${ }^{82} \mathrm{Rb}$ (panel B). The red data points highlight the sensitivity/ specificity pairs reported by Juneau et al. The probability of achieving or exceeding the reported sensitivity, specificity, and area under the curve of the receiver-operating characteristic plot is only $0.97 \%$ for ${ }^{13} \mathrm{NH}_{3}$ and $0.18 \%$ for ${ }^{82} \mathrm{Rb}$, respectively.

the simulations are available in the electronic supplementary material]. Based on our simulations, real-world sensitivities are likely to be approximately $65.2 \%$ and $64.2 \%$, respectively, for ${ }^{13} \mathrm{NH}_{3}$ and ${ }^{82} \mathrm{Rb}$, markedly lower than reported and insufficient for clinical use. Conversely, real-world specificities for ${ }^{13} \mathrm{NH}_{3}$ and ${ }^{82} \mathrm{Rb}$ are likely to be $78.0 \%$ and $86.0 \%$, respectively, closer to the reported values (Figure 2). These simulated results suggest that there may have been adjustment of falsenegative studies, thus, improving the reported sensitivity. The real-world AUCs are also likely to underperform at 0.798 and 0.854 for ${ }^{13} \mathrm{NH}_{3}$ and ${ }^{82} \mathrm{Rb}$, respectively. If one assumes that the ${ }^{13} \mathrm{NH}_{3}$ and ${ }^{82} \mathrm{Rb}$ experiments are independent, given the distribution of MFR, estimated MFR, and their correlations, the probability of obtaining results as good as reported for sensitivity, specificity, and AUC for both is less than 1 in $50000(P=0.000017)$.

As a result, while the study shows promise, it is likely that in clinical practice, this method will not reliably reproduce MFR, yielding limits of agreement far too wide for clinical use. The method seems more applicable as a quality control tool, as discrepancies between the estimated and standard MFR larger than the limits of agreement may indicate some issue with one of the two calculations. However, even this role is potentially limited by the assumption of unchanged $\mathrm{CO}$ between rest and stress. Furthermore, when a discrepancy is found, it may be challenging to identify which of the two measures is reliable and the reasons to discount the other.

\section{WAKING UP FROM THE DREAM}

Ultimately, the work put forth by Juneau et al is of uncertain value toward simplifying MFR quantification. The clinical implementation of this method requires rigorous blinded validation to prove incremental value over relative perfusion or value as a quality control tool. To be clear, the likely adjustment of false-negative studies does not constitute unethical research behavior. Indeed, it fits with standard clinical behavior wherein physicians will remeasure or reprocess studies which do not meet their expectations. However, when this is done selectively, without blinding to the gold standard, severe biases may result. ${ }^{17}$ Therefore, unless this study is repeated with independent blinded core labs for separately processing the dynamic and static images, it is not possible to conclude that this method is ready for prime time.

A few other limitations should be considered. First, the application of the method to ${ }^{13} \mathrm{NH}_{3}$ only makes sense for exercise stress, given that saturation is rarely an issue for that tracer, even on older scanners. However, exercise testing is likely to introduce markedly greater variability in the $\mathrm{CO}$ with a much larger increase in $\mathrm{CO}$ 
from rest to stress than with dipyridamole. Consequently, this method would require further validation, relying on a different gold standard than traditional MFR given that it is nearly impossible to collect highquality dynamic images during exercise stress.

Although a tool to detect saturation for ${ }^{82} \mathrm{Rb}$ MFR would be of tremendous value, the results provided stop short of demonstrating that the method works to identify saturation in this study. Indeed, the effect of saturation on MFR has yet to be fully characterized in the literature, in part due to the lack of a simple, widely accepted gold standard for saturation and for the tissue time-activity curve.

In the end, this study does offer a valuable lesson for clinical researchers. Blinding is necessary to ensure that results are not inadvertently biased while intending to maximize quality. Hopefully this work can be a steppingstone toward greater reliability, accessibility, and ease of clinical MFR assessment.

\section{Disclosures}

A. Poitrasson-Rivière is an employee of INVIA Medical Imaging Solutions. V.L. Murthy is supported by R01AG059729 from the National Institute on Aging, U01DK123013 from the National Institute of Diabetes and Digestive and Kidney Disease, and R01HL136685 from the National Heart, Lung, and Blood Institute as well as the Melvyn Rubenfire Professorship in Preventive Cardiology. Dr. Murthy has received research grants and speaking honoraria from Siemens Medical Imaging. He serves as a scientific advisor for Ionetix and owns stock options in the same. Dr. Murthy also owns stock in General Electric and Cardinal Health. He has received expert witness payments on behalf of Jubilant Draximage and a speaking honorarium from 2Quart Medical. Dr. Murthy receives non-financial research support from INVIA Medical Imaging Solutions.

\section{References}

1. Naya M, Murthy VL, Taqueti VR, Foster CR, Klein J, Garber M, et al. Preserved coronary flow reserve effectively excludes highrisk coronary artery disease on angiography. J Nucl Med 2014;55:248-55. https://doi.org/10.2967/jnumed.113.121442.

2. Murthy VL, Naya M, Foster CR, Hainer J, Gaber M, Carli G, et al. Improved cardiac risk assessment with noninvasive measures of coronary flow reserve. Circulation 2011;124:2215-24. https://doi. org/10.1161/CIRCULATIONAHA.111.050427.

3. Gould KL, Johnson NP, Roby AE, Nguyen T, Kirkeeide R, Haynie $\mathrm{M}$, et al. Regional, artery-specific thresholds of quantitative myocardial perfusion by PET associated with reduced myocardial infarction and death after revascularization in stable coronary artery disease. J Nucl Med 2019;60:410-7. https://doi.org/10.2967/ jnumed.118.211953.

4. Murthy VL, Bateman TM, Beanlands RS, Berman DS, BorgesNeto $\mathrm{S}$, Chareonthaitawee $\mathrm{P}$, et al. Clinical quantification of myocardial blood flow using PET: Joint position paper of the SNMMI cardiovascular council and the ASNC. J Nucl Cardiol 2017. https://doi.org/10.1007/s12350-017-1110-x.

5. Lee BC, Moody JB, Poitrasson-Rivière A, Melvin AC, Weinberg RL, Corbett JR, et al. Blood pool and tissue phase patient motion effects on ${ }^{82}$ rubidium PET myocardial blood flow quantification. $\mathbf{J}$ Nucl Cardiol 2018. https://doi.org/10.1007/s12350-018-1256-1.

6. Armstrong IS, Memmott MJ, Saint KJ, Saillant A, Hayden C, Arumugam P, et al. Assessment of motion correction in dynamic rubidium-82 cardiac PET with and without frame-by-frame adjustment of attenuation maps for calculation of myocardial blood flow. J Nucl Cardiol 2019. https://doi.org/10.1007/s12350019-01834-5.

7. Poitrasson-Rivière A, Moody JB, Hagio T, Weinberg RL, Corbett JR, Murthy VL, et al. Reducing motion-correction-induced variability in ${ }^{82}$ rubidium myocardial blood-flow quantification. J Nucl Cardiol 2020;27:1104-13. https://doi.org/10.1007/s12350-019-01 911-9.

8. Renaud JM, Yip K, Guimond J, Trottier M, Pibarot P, Turcotte E, et al. Characterization of 3-dimensional PET systems for accurate quantification of myocardial blood flow. J Nucl Med 2016;58:10310. https://doi.org/10.2967/jnumed.116.174565.

9. Yoshida K, Mullani N, Gould KL. Coronary flow and flow reserve by PET simplified for clinical applications using rubidium-82 or nitrogen-13-ammonia. J Nucl Med 1996;37:1701-12.

10. Sherif HM, Nekolla SG, Saraste A, Reder S, Yu M, Robinson S, et al. Simplified quantification of myocardial flow reserve with flurpiridaz F 18: Validation with microspheres in a pig model. J Nucl Med 2011;52:617-24. https://doi.org/10.2967/jnumed.110. 083196.

11. Juneau D, Wu KY, Kaps N, Yao J, Renaud JM, Beanlands RSB, et al. Internal validation of myocardial flow reserve PET imaging using stress/rest myocardial activity ratios with $\mathrm{Rb}-82$ and N-13ammonia. J Nucl Cardiol 2021. https://doi.org/10.1007/s12350-0 20-02464-y.

12. Bellina CR, Parodi O, Camici P, Salvadori PA, Taddei L, Fusani $\mathrm{L}$, et al. Simultaneous in vitro and in vivo validation of nitrogen13-ammonia for the assessment of regional myocardial blood flow. J Nucl Med 1990;31:1335-43.

13. Dorbala S, Hachamovitch R, Curillova Z, Thomas D, Vangala D, Kwong RY, et al. Incremental prognostic value of gated $\mathrm{Rb}-82$ positron emission tomography myocardial perfusion imaging over clinical variables and rest LVEF. JACC Cardiovasc Imaging 2009;2:846-54. https://doi.org/10.1016/j.jcmg.2009.04.009.

14. Hage FG, Dean P, Iqbal F, Heo J, Iskandrian AE, et al. A blunted heart rate response to regadenoson is an independent prognostic indicator in patients undergoing myocardial perfusion imaging. $\mathbf{J}$ Nucl Cardiol 2011;18:1086-94. https://doi.org/10.1007/s12350-01 1-9429-1.

15. Lskandrian AS, Verani MS, Heo J. Pharmacologic stress testing: Mechanism of action, hemodynamic responses, and results in detection of coronary artery disease. J Nucl Cardiol 1994;1:94111.

16. Shun-Shin MJ, Francis DP. Why even more clinical research studies may be false: Effect of asymmetrical handling of clinically unexpected values. PLoS ONE 2013. https://doi.org/10.1371/jour nal.pone. 0065323 .

17. Ioannidis JP, Haidich AB, Pappa M, Pantazis N, Kokori SI, Tektonidou MG, et al. Comparison of evidence of treatment effects in randomized and nonrandomized studies. J Am Med Assoc 2001;286:821-30. https://doi.org/10.1001/jama.286.7.821.

Publisher's Note Springer Nature remains neutral with regard to jurisdictional claims in published maps and institutional affiliations. 